\title{
Algorithms and Strategies for Treatment of Large Deformation Frictional Contact in the Numerical Simulation of Deep Drawing Process
}

\author{
M.C. Oliveira · J.L. Alves $\cdot$ L.F. Menezes
}

Received: 9 March 2007 / Accepted: 9 March 2007 / Published online: 14 February 2008

(C) CIMNE, Barcelona, Spain 2008

\begin{abstract}
This paper describes a fully implicit algorithm developed and optimized to simulate sheet metal forming processes. This algorithm was implemented in the inhouse code DD3IMP. Attention is paid to the augmented lagrangian method adopted to treat the contact with friction problem. The global resolution of the coupled equilibrium and contact problem is performed in a single loop, with a static implicit iterative Newton-Raphson scheme. This demands particular attention in the contact search algorithm, which in this case adopts a parametric description of the tools. In order to highlight the adopted strategies a review of the state-of-the-art in sheet metal forming simulation is presented, with respect to models reliability and efficiency.
\end{abstract}

\section{Introduction}

The virtual try-out of sheet metal forming components based on the finite element method has become an indispensable industrial tool in order to save money, time and effort in the design, process set-up and production of deep drawn parts. The importance of the numerical simulation in the shortening of the conception cycles of new deep drawn parts is unquestionable, since it allows the optimisation of the process parameters involved, like the lubrication conditions, the prediction of the required tools force (punch, blank holder and

M.C. Oliveira $(\varangle) \cdot$ L.F. Menezes

Department of Mechanical Engineering, University of Coimbra,

Polo II, Rua Luís Reis Santos, Pinhal de Marrocos, 3030-788

Coimbra, Portugal

e-mail: marta.oliveira@dem.uc.pt

J.L. Alves

Department of Mechanical Engineering, University of Minho, Campus de Azurém, 4800-058 Guimarães, Portugal die), the gap, etc. Also, according with the chosen material for the blank it is possible to perform a direct comparison of process parameters' behaviour.

Due to its enormous economical impact, the application of the finite element method to numerical simulation of deep drawing processes has suffered many improvements in the last years. However, there are still old and new challenges. To achieve the complete virtual try-out of a component it is essential to accurately predict forming defects, in particular geometrical defects associated with springback phenomena and wrinkling. Other challenge is to accurately simulate multi-stage processes. Reliable multi-stage forming simulations are only possible with an accurate description of technological procedure and parameters, and if material's mechanical behaviour is well understood. Much of the recent research and development effort in the numerical simulation of sheet metal forming has focused on these two aspects, in order to improve accuracy of numerical codes. In fact, if these aspects drive the accuracy in single-stage forming processes, they become of paramount importance in multistage forming processes; since errors play a cumulative role and inaccuracy in former stages are amplified in subsequent ones.

The majority of the research areas involved in numerical simulation of deep drawing processes are still under development in order to increase accuracy and robustness of numerical codes, and reduce computational time and memory requirements. In this context, the contact with friction problem is a very interesting topic. The contact between the tools and the blank determines the evolutionary boundary conditions of the forming process. Thus, it is essential to model accurately such phenomenon in order to assure the final accuracy of numerical results. Due to the strong non-linearity of the contact with friction problem, solve this kind of problems with the finite element method presents many difficul- 